\title{
THE CONSTRUCTION AND SMOOTHNESS OF INVARIANT MANIFOLDS BY THE DEFORMATION METHOD*
}

\author{
JERROLD MARSDEN $\dagger$ AND JÜRGEN SCHEURLE
}

\begin{abstract}
This paper proves optimal results for the invariant manifold theorems near a fixed point for a mapping (or a differential equation) by using the deformation, or Lie transform, method from singularity theory. The method was inspired by the difficulties encountered by the implicit function theorem technique in the case of the center manifold. The idea here is simply to deform the given system into its linearization and to track this deformation using the flow of a time-dependent vector field. Corresponding to the difficulties with the center manifold encountered by other techniques, we run into a "derivative loss" in this case as well, which is overcome by utilizing estimates on the differentiated equation. A survey of the other methods used in the literature is also presented.
\end{abstract}

Key words. invariant manifold, deformation method, center manifold, Lie transform

AMS(MOS) subject classification. 58F

1. Introduction. The theory of invariant manifolds is one of the fundamental ingredients in the study of dynamical systems. In this theory one looks for submanifolds of the phase space which are invariant under the flow, i.e., trajectories which start on such a manifold at some time, stay on it.

This problem is not only of interest from a qualitative point of view, but can lead to quantitative results. In fact, by restriction to an invariant manifold, an original system is reduced to a lower-dimensional one which might be relatively simple. In particular, this is the case when the phase space of the original system is infinitedimensional and one considers finite-dimensional invariant submanifolds. An important example for applications is the center manifold which contains all bounded solutions near a fixed point [3], [15], [16].

The well-known invariant manifold theorems refer to the flow generated by a nonlinear vector field or diffeomorphism defined in a neighborhood of a fixed point $[8],[10],[11],[14]$. They give sufficient conditions for the existence of an invariant submanifold which contains this fixed point. For example, each component of the spectral decomposition of the phase space corresponding to a linear operator is an invariant subspace for the flow generated by this linear operator. In the general nonlinear theory one begins with such an invariant subspace of the linearized system and shows its persistence as an invariant submanifold for the full system (at least locally) and then one determines the smoothness of the resulting nonlinear manifold ([6]; cf. also [9]).

To construct such invariant manifolds, two different approaches have been used in the literature so far. First, the invariance property of the manifold has been used to derive an equation for a representing map [10], [11], [14]. The manifold is sought as a graph and an iteration scheme is used on the graphs. For diffeomorphisms, this "graph transform method" developed in [11] yields optimal results and even holds for "Lipeomorphisms" (also [18] and [20]). Second, asymptotic properties of the flow on the manifold have been used to derive an equation for the corresponding trajectories $[2],[5],[7],[8],[12]$. All these trajectories together span the desired manifold and

* Received by the editors May 9, 1986; accepted for publication (in revised form) August 14, 1986.

tDepartment of Mathematics, University of California, Berkeley, California 94720 . The research of this author was partially supported by National Science Foundation contract DMS 84-04506.

$\ddagger$ Department of Mathematics, Colorado State University, Fort Collins, Colorado 80523, and Mathematisches Institut A der Universität Stuttgart, Pfaffenwaldring 57, D-7000 Stuttgart 80, West Germany. 
invariance is a consequence. Alternatively, this second approach may be phrased as a fixed point problem for a map representing the manifold by considering the initial values of the trajectories parameterized over the invariant subspace of the linearized system [4], [19]. In both cases in the second approach, the resulting equation may be solved iteratively. For stable and unstable invariant manifolds the equation for the trajectories also has been solved using the classical implicit function theorem [12]. This yields optimal smoothness for $C^{k}$ vector fields and diffeomorphisms, and even in the analytic case.

Unfortunately, it is not obvious how to apply the classical implicit function theorem for general invariant manifolds, e.g., for center manifolds. In general, the operator underlying the equation for the trajectories is not continuously differentiable in a space of functions which have the right asymptotic behavior (exponential growth). This difficulty always occurs for the equation of a representing map in a space of maps with a certain smoothness. Sacker [17] uses a smoothing technique to overcome this difficulty, but he still loses one order of smoothness for the solution. For unsuccessful attempts to apply the implicit function theorem in the case of center manifolds, see [4] and [13].

In the present paper we solve the equation for a representing map using a different approach, namely the "Lie transform" method of integrating a differential equation which is based on a deformation principle. This method has been used for the Darboux theorem, the Frobenius theorem and the Poincaré lemma [1] and is a common tool in singularity theory. The idea is to consider a one-parameter family of systems connecting the given system with its linearization. Differentiation with respect to the parameter yields a linear equation for a vector field which eventually has to be integrated in order to get the desired map. An initial condition is known from the invariant manifold of the linearized system.

We consider only diffeomorphisms here, although a similar approach for vector fields is possible. Our approach applies for general invariant manifolds; although we shall concentrate on the harder case of center manifolds, we indicate how results about other invariant manifolds can be obtained. Our smoothness results are optimal. We note at the outset that the diffeomorphisms which we are going to consider have to be of class $C_{\text {Lip }}^{3}$ at least. This is the price we pay for our more sophisticated method.

The plan of the paper is as follows. In $\$ 2$ we state our main results. Theorem 2.1 is an existence and uniqueness result for a global center stable or center unstable invariant manifold of a $C_{\text {Lip }}^{3}$ map in a Banach space. Corollary 2.2 contains the corresponding smoothness result for $C^{k}(k \geqq 4)$ and $C_{\text {Lip }}^{k}(k \geqq 3)$ maps. In Remark 2.3 we list certain modifications and generalizations of these results. Finally, $\$ \$ 3$ and 4 contain the proofs of Theorem 2.1 and Corollary 2.2.

2. Formulation of the problem and results. Let $X$ and $Y$ be Banach spaces. The product space is denoted by $X \times Y$ and equipped with the sup-norm. The Banach space of $k$-linear continuous maps from $X$ to $Y$ equipped with the usual norm induced by the norms of $X$ and $Y$ is denoted by $\mathscr{L}^{k}(X, Y)$, and we let $\mathscr{L}^{k}(X, X)=\mathscr{L}^{k}(X)$.

Also we introduce the Banach space $C^{k}(V, Y)$ of $k$-times continuously differentiable maps $f$ from an open subset $V \subset X$ into $Y$, equipped with the norm

$$
\|f\|_{k}=\sup _{\substack{x \in V \\ 0 \leq i \leq k}}\left\|D^{i} f(x)\right\|
$$

where $D^{i} f$ denotes the $i$ th derivative of $f$. Similar to the above, we set $C^{k}(X, X)=$ $C^{k}(X)$. The linear subspace of those elements of $C^{k}(V, Y)$ for which the $k$ th derivative 
is Lipschitz continuous in $V$ is denoted by $C_{\mathrm{Lip}}^{k}(V, Y)$. Furthermore, we introduce the notation $C_{L}^{k}(V, Y)$ for the closed subset of elements of $C^{k}(V, Y)$ which satisfy a Lipschitz condition in $V$ with a particular Lipschitz constant $L$.

Open balls in Banach spaces are denoted by $B_{r}(\cdot)$, where $r$ is the radius and the point stands for the center of the ball. The closure of a set $V$ is written as $\mathrm{cl}(V)$.

Let us consider a map

$$
T: X \times Y \rightarrow X \times Y, \quad(x, y) \mapsto\left(\phi_{1}(x, y), \phi_{2}(x, y)\right)
$$

given by

$$
\phi_{1}(x, y)=A x+f(x, y), \quad \phi_{2}(x, y)=B y+g(x, y),
$$

where $A \in \mathscr{L}(X), B \in \mathscr{L}(Y)$, and $f$ and $g$ are (nonlinear) perturbations. We consider the following hypotheses, for $\delta \in \mathbb{R}^{+}$and $k$ an integer:

$(\mathrm{L} 1)_{k} \quad\left\|B^{-1}\right\|\left\|A^{j}\right\|<1 \quad$ for $0 \leqq j \leqq k$.

$(\mathrm{L} 2)_{k} \quad\|B\|\left\|A^{-j}\right\|<1 \quad$ for $0 \leqq j \leqq k$.

(N1) $\delta \in C_{\mathrm{Lip}}^{3}(X \times U, X)$, and $g \in C_{\mathrm{Lip}}^{3}(X \times U, Y)$ where $U$ is some neighborhood of 0 in $Y$;

$\|f\|_{1}<\delta$ and $\|g\|_{1}<\delta$.

(N2) $f(0,0)=0, \quad g(0,0)=0$.

(N3) $\quad D_{x} g(0,0)=0$.

Note that $(0,0)$ is a fixed point of $T$ when (N2) holds.

We shall prove the following theorem about a so-called center stable or center unstable manifold.

TheOREM 2.1. Let the assumption (L1) ${ }_{4}$ or $(\mathrm{L} 2)_{4}$, and $(\mathrm{N} 1)_{\delta}$ hold, where $\delta>0$ is sufficiently small. Then there is a map $h \in C_{\text {Lip }}^{3}(X, Y)$ with $\|h\|_{1}=O(\delta)$ as $\delta \rightarrow 0$, such that the manifold

$$
M=\{(x, y) \in X \times Y \mid y=h(x)\}
$$

is invariant under the iteration of the map $T$, i.e., $(x, y) \in M$ implies $T(x, y) \in M$; the map $h$ is unique in $C_{L}(X, Y)$, where $L=O(1 / \delta)$ as $\delta \rightarrow 0$.

If in addition (N2) (resp. (N2) and (N3)) hold, then $h(0)=0$ (resp. $h(0)=0$ and $\left.D_{x} h(0)=0\right)$.

COROLlary 2.2. Assume that $f$ and $g$ are of class $C_{\mathrm{Lip}}^{k-1}\left(\right.$ resp. $\left.C^{k}\right)$ for some $k \geqq 4$. Furthermore, let (L1) ${ }_{k}$ or $(\mathrm{L} 2)_{k}$, and $(\mathrm{N} 1)_{\delta}$ hold. Then $h$ is of class $C_{\text {Lip }}^{k-1}$ (resp. $C^{k}$ ) provided that $\delta$ is sufficiently small. (In general $\delta$ depends on $k$ for given $A$ and $B$.)

In the following remark we state some generalizations and modifications of the above results, which are obvious from the proofs in the next sections.

Remarks 2.3. (i) If $B$ decomposes into two parts $B_{1}$ and $B_{2}$ such that $B_{1}$ satisfies $(\mathrm{L} 1)_{k}$ and $B_{2}$ satisfies (L2) ${ }_{k}$ for some $k \geqq 4$, then the above assertions remain true. In this case $M$ is called a center manifold.

(ii) If $\left\|B^{-1}\right\|<1$ and $\|A\|<1$ (resp. $\|B\|<1$ and $\left\|A^{-1}\right\|<1$ ), then $M$ is called the stable (resp. unstable) invariant manifold. In this case $M$ is a $C^{\infty}$ manifold if $f$ and $g$ are of class $C^{\infty}$. (Here $\delta$ does not depend on $k$.) Moreover, in this case $M$ is even analytic for analytic maps $f$ and $g$. For the stable manifold this follows by using spaces of complex analytic functions instead of $C_{\text {Lip }}^{1}$ functions in the existence proof. The unstable manifold case is reduced to the stable one just by considering the map $T^{-1}$ instead of $T$, provided that it exists. 
(iii) If the assumptions are only fulfilled when $x$ is restricted to some neighborhood of 0 in $X$, then one can use a cut-off function $\chi: X \rightarrow \mathbb{R}$ to extend $f$ and $g$ to the domain $X \times U$. This is a $C^{\infty}$ function with the property $\chi(x)=1$ for $\|x\| \leqq \frac{1}{2}$ and $\chi(x)=0$ for $\|x\| \geqq 1$. Such a function always exists if $X$ is finite-dimensional. The extensions are given by $\tilde{f}(x, y)=f(\chi(\mu x) x, y)$ and $\tilde{g}(x, y)=g(\chi(\mu x) x, y)$ with an appropriate constant $\mu>0$. Applying our results for $\tilde{f}$ and $\tilde{g}$ then yields a local invariant manifold for the original map $T$ by restricting $h$ to the ball $\|x\|<\mu^{-1} / 2$.

This cut-off procedure destroys uniqueness and analyticity for the local case. On the other hand, we do not need the cut-off procedure for the local theory when $\|A\|<1$ (or $\left\|A^{-1}\right\|<1$ ). In that case we can directly work with spaces of maps which are defined only in some ball around $x=0$. This yields local results for general spaces $X$ and, in particular, analyticity. Hence, local stable (unstable) invariant manifolds are analytic if $f$ and $g$ are analytic. Furthermore, under the additional hypothesis that $f$ and $g$ together with all partial derivatives of $g$ with respect to $x$ up through order $l-1$ vanish at $(0,0)$, the local results still hold when $\|A\|<1$ (or $\left\|A^{-1}\right\|<1$ ) and the inequalities in ( $(\mathrm{L} 1)_{k}\left((\mathrm{~L} 2)_{k}\right)$ only hold for $l \leqq j \leqq k$ for some $l \geqq 1$. In this case one has to work with functions $h$ and $H=D_{x} h$ which have the properties $\|h(x)\| \leqq C_{1}\|x\|^{l},\|D h(x)\| \leqq$ $C_{2}\|x\|^{l-1},\|H(x)\| \leqq C_{3}\|x\|^{I-1}$ and $\|D H(x)\| \leqq C_{4}\|x\|^{l-2}$ in some ball around $x=0$ with certain constants $C_{j}$. It finally follows that $\left\|D^{j} h(x)\right\| \leqq C_{j}\|x\|^{l-j}$ for $0 \leqq j \leqq l-1$. Note that strong, stable (or unstable) invariant manifolds, where $l=1$, and also certain weak stable (or unstable) invariant manifolds are included in this local theory.

(iv) To obtain a smoothness result for $M$ with respect to a parameter $\lambda \in \Lambda$, where $\Lambda$ is some Banach space, we can consider $\lambda$ as a component of $x$ by adding the trivial component $\lambda \mapsto \lambda$ to the original map $T$ (cf., [15]).

(v) Theorem 2.1 and Corollary 2.2 remain true if, in the definition of $T$, the terms $A x$ and $B y$ are replaced by any maps $A(x): X \rightarrow X$ and $B(x) y: X \times Y \rightarrow Y$ which are as smooth as $f$ and $g$ and satisfy the following assumptions:

$$
\begin{array}{ll}
\left\|B(x)^{-1}\right\|\left\|D A(x)^{j}\right\|<1 & \text { for } 0 \leqq j \leqq k, \\
\|B(x)\|\left\|D A^{-1}(x)^{j}\right\|<1 & \text { for } 0 \leqq j \leqq k .
\end{array}
$$

For example, this generalization is relevant when one deals with a suspension of a nonautonomous system in the extended phase space which is the product of the (discrete) time axis and the original phase space.

(vi) Finally, we remark that it suffices to require $\left\|D_{x} f\right\|<\delta$ instead of $\|D f\|<\delta$ to prove the above results.

3. Proof of Theorem 2.1. We begin with the existence part. First we outline the basic ideas of our proof in a more or less formal way. Afterwards we shall justify each step by means of a series of lemmas.

We consider the following one-parameter family of maps:

$$
T_{\varepsilon}: X \times Y \rightarrow X \times Y, \quad(x, y) \mapsto\left(\phi_{1}(\varepsilon, x, y), \phi_{2}(\varepsilon, x, y)\right)
$$

given by

$$
\phi_{1}(\varepsilon, x, y)=A x+\varepsilon f(x, y), \quad \phi_{2}(\varepsilon, x, y)=B y+\varepsilon g(x, y),
$$

for $\varepsilon$ a real number. Obviously $T_{\varepsilon}$ defines a homotopy between the linear map

$$
T_{0}:(x, y) \mapsto(A x, B y) \text { and } T_{1}=T \text {. }
$$

For each $T_{\varepsilon}$ we are looking for an invariant manifold $M_{\varepsilon}$ of the form $y=h_{\varepsilon}(x)$, where the map $h_{\varepsilon}: X \rightarrow Y$ depends smoothly on $\varepsilon$. The invariance property leads to 
the equation

$$
h_{\varepsilon}\left(\phi_{1}\left(\varepsilon, x, h_{\varepsilon}(x)\right)\right)=\phi_{2}\left(\varepsilon, x, h_{\varepsilon}(x)\right)
$$

Moreover, we require

$$
h_{0}(x)=0 \text { for } x \in X,
$$

since $y=0$ is an invariant manifold of $T_{0}$. Thus, we aim to solve the system of equations (3.2) and (3.3) for $h_{\varepsilon}(x)$.

The main idea now is to derive a first order differential equation for the function $\varepsilon \mapsto h_{\varepsilon}$ and to integrate this in the interval $0 \leqq \varepsilon \leqq 1$ with (3.3) as an initial condition. Actually, we shall consider a differential system for the function $\varepsilon \mapsto\left(h_{\varepsilon}, H_{\varepsilon}\right)$, where

$$
H_{\varepsilon}(x)=D_{x} h_{\varepsilon}(x) \in \mathscr{L}(X, Y)
$$

Thus we get a linear equation for the corresponding vector field which can be solved explicitly. Subsequently the arguments of $\phi_{1}, \phi_{2}$ and all their derivatives are $\left(\varepsilon, \cdot, h_{\varepsilon}(\cdot)\right)$, if not indicated otherwise. A dot above a symbol for a map denotes the partial derivative with respect to $\varepsilon$.

First we differentiate equation (3.2) with respect to $\varepsilon$, which yields

$$
\dot{h}_{\varepsilon}\left(\phi_{1}\right)-\mathscr{B} \dot{h}_{\varepsilon}=\mathscr{F}_{1},
$$

where

$$
\mathscr{B}=\mathscr{B}\left(\varepsilon, h_{\varepsilon}, H_{\varepsilon}\right)=D_{y} \phi_{2}-H_{\varepsilon}\left(\phi_{1}\right) D_{y} \phi_{1}
$$

is a map from $X$ to $\mathscr{L}(Y)$ and

$$
\mathscr{F}_{1}=\mathscr{F}_{1}\left(\varepsilon, h_{\varepsilon}, H_{\varepsilon}\right)=\dot{\phi}_{2}-H_{\varepsilon}\left(\phi_{1}\right) \dot{\phi}_{1}
$$

is a map from $X$ to $Y$. An equation for $H_{\varepsilon}$ is obtained by differentiating (3.2) with respect to $x$. Thus we obtain

$$
H_{\varepsilon}\left(\phi_{1}\right) \mathscr{A}-D_{y} \phi_{2} H_{\varepsilon}=D_{x} \phi_{2}
$$

where

$$
\mathscr{A}=\mathscr{A}\left(\varepsilon, h_{\varepsilon}, H_{\varepsilon}\right)=D_{x} \phi_{1}+D_{y} \phi_{1} H_{\varepsilon}
$$

is a map from $X$ to $\mathscr{L}(X, Y)$. Since this equation is still nonlinear, we again differentiate it with respect to $\varepsilon$. Setting

$$
G_{\varepsilon}(x)=D_{x} H_{\varepsilon}(x) \in \mathscr{L}^{2}(X, Y),
$$

we thus get

$$
\dot{H}_{\varepsilon}\left(\phi_{1}\right) \mathscr{A}-\mathscr{B} \dot{H}_{\varepsilon}+\mathscr{C} \dot{h}_{\varepsilon}=\mathscr{F}_{2}
$$

where

$$
\begin{aligned}
\mathscr{C} \dot{h}_{\varepsilon}= & \mathscr{C}\left(\varepsilon, h_{\varepsilon}, H_{\varepsilon}, G_{\varepsilon}\right) \dot{h}_{\varepsilon} \\
= & G_{\varepsilon}\left(\phi_{1}\right)\left(\mathscr{A}, D_{y} \phi_{1} \dot{h}_{\varepsilon}\right)+H_{\varepsilon}\left(\phi_{1}\right)\left(D_{x y}^{2} \phi_{1} \dot{h}_{\varepsilon}+D_{y y}^{2} \phi_{1}\left(H_{\varepsilon}, \dot{h}_{\varepsilon}\right)\right) \\
& -D_{x y}^{2} \phi_{2}\left(\cdot, \dot{h}_{\varepsilon}\right)-D_{y y}^{2} \phi_{2}\left(H_{\varepsilon}, \dot{h}_{\varepsilon}\right),
\end{aligned}
$$




$$
\begin{aligned}
\mathscr{F}_{2} & =\mathscr{F}_{2}\left(\varepsilon, h_{\varepsilon}, H_{\varepsilon}, G_{\varepsilon}\right) \\
& =D_{x} \dot{\phi}_{2}+D_{y} \dot{\phi}_{2} H_{\varepsilon}-G_{\varepsilon}\left(\phi_{1}\right)\left(\mathscr{A}, \dot{\phi}_{1}\right)-H_{\varepsilon}\left(\phi_{1}\right)\left(D_{x} \dot{\phi}_{1}+D_{y} \dot{\phi}_{1} H_{\varepsilon}\right)
\end{aligned}
$$

are maps from $X$ to $\mathscr{L}(X, Y)$. A linear equation for $G_{\varepsilon}$ is obtained by differentiating (3.6) with respect to $x$ and using (3.4) again

$$
G_{\varepsilon}\left(\phi_{1}\right)(\mathscr{A}, \mathscr{A})-\mathscr{B} G_{\varepsilon}=\mathscr{F}_{3}
$$

where

$$
\begin{aligned}
\mathscr{F}_{3}=\mathscr{F}_{3}\left(\varepsilon, h_{\varepsilon}, H_{\varepsilon}\right)=D_{x x}^{2} \phi_{2} & +2 D_{x y}^{2} \phi_{2}\left(\cdot, H_{\varepsilon}\right)+D_{y y}^{2} \phi_{2}\left(H_{\varepsilon}, H_{\varepsilon}\right) \\
& -H_{\varepsilon}\left(\phi_{1}\right)\left(D_{x x}^{2} \phi_{1}+2 D_{x y}^{2} \phi_{1}\left(\cdot, H_{\varepsilon}\right)+D_{y y}^{2} \phi_{2}\left(H_{\varepsilon}, H_{\varepsilon}\right)\right)
\end{aligned}
$$

is a map from $X$ to $\mathscr{L}^{2}(X, Y)$.

Now we proceed as follows. For each fixed real $\varepsilon$ and for each fixed pair of maps $h_{\varepsilon}: X \rightarrow Y$ and $H_{\varepsilon}: X \rightarrow \mathscr{L}(X, Y)$, we solve (3.9) for $G_{\varepsilon}$. The solution is written in the form

$$
G_{\varepsilon}=\mathscr{G}\left(\varepsilon, h_{\varepsilon}, H_{\varepsilon}\right)
$$

where $\mathscr{G}\left(\varepsilon, h_{\varepsilon}, H_{\varepsilon}\right)$ is a map from $X$ to $\mathscr{L}^{2}(X, Y)$. Inserting this expression into (3.8), we obtain

$$
\dot{H}_{\varepsilon}\left(\phi_{1}\right) \mathscr{A}-\mathscr{B} \dot{H}_{\varepsilon}+\mathscr{C}\left(\varepsilon, h_{\varepsilon}, H_{\varepsilon}, \mathscr{G}\left(\varepsilon, h_{\varepsilon}, H_{\varepsilon}\right)\right) \dot{h}_{\varepsilon}=\mathscr{F}_{2}\left(\varepsilon, h_{\varepsilon}, H_{\varepsilon}, \mathscr{G}\left(\varepsilon, h_{\varepsilon}, H_{\varepsilon}\right)\right) .
$$

This relation together with (3.5) is linear equation for $\left(\dot{h}_{\varepsilon}, \dot{H}_{\varepsilon}\right)$, which we write as

$$
\left(\begin{array}{c}
\dot{h}_{\varepsilon} \\
\dot{H}_{\varepsilon}
\end{array}\right)=\mathscr{H}\left(\varepsilon, h_{\varepsilon}, H_{\varepsilon}\right)
$$

where the right-hand side is a map from $X$ to $Y \times \mathscr{L}(X, Y)$. This is the desired differential equation.

By the derivation of this equation, every two times continuously differentiable function $h_{\varepsilon}(x)$ which satisfies (3.2), together with its partial derivative $H_{\varepsilon}(x)=D_{x} h_{\varepsilon}(x)$, is a solution. To show that vice versa a solution $\left(h_{\varepsilon}(x), H_{\varepsilon}(x)\right)$ of (3.12) such that (3.3) and

$$
H_{0}(x)=0 \quad \text { for } x \in X
$$

are satisfied yields a solution of (3.2), we show that $H_{\varepsilon}$ is actually the partial derivative of $h_{\varepsilon}$ with respect to $x$, i.e., (3.4) is satisfied. Inserting (3.4) into (3.5) and integrating with respect to $\varepsilon$, we then get relation (3.2). Here we use the fact that by $(3.3), h_{\varepsilon}(x)$ solves (3.2) for $\varepsilon=0$.

To prove (3.4), we differentiate (3.5) and (3.8) with respect to $x$, which gives

$$
\begin{aligned}
& \dot{p}_{j}\left(\phi_{1}\right) \mathscr{A}\left(\varepsilon, h_{\varepsilon}, p_{j}\right)-\mathscr{B} \dot{p}_{j}=\mathscr{F}_{4}\left(\varepsilon, p_{j}, q_{j}\right), \\
& \dot{q}_{j}\left(\phi_{1}\right)\left(\mathscr{A}\left(\varepsilon, h_{\varepsilon}, p_{j}\right), \mathscr{A}\right)-\mathscr{B} \dot{q}_{j}+\mathscr{C} \dot{p}_{j}=\mathscr{F}_{5}\left(\varepsilon, p_{j}, q_{j}, q_{l(j)}\right),
\end{aligned}
$$

for $j=1$, where

$$
l(1)=2, \quad p_{1}=D_{x} h_{\varepsilon}, \quad q_{1}=D_{x} H_{\varepsilon}, \quad q_{2}=G_{\varepsilon},
$$




$$
\begin{aligned}
\mathscr{F}_{4}\left(\varepsilon, p_{j}, q_{j}\right)=D_{x} \dot{\phi}_{2} & +D_{y} \dot{\phi}_{2} p_{j}+D_{y x}^{2} \phi_{2}\left(\dot{h}_{\varepsilon}, \cdot\right)+D_{y y}^{2} \phi_{2}\left(p_{j}, \dot{h}_{\varepsilon}\right) \\
& -q_{j}\left(\phi_{1}\right)\left(\mathscr{A}\left(\varepsilon, h_{\varepsilon}, p_{j}\right),\left(\dot{\phi}_{1}+D_{y} \phi_{1} \dot{h}_{\varepsilon}\right)\right) \\
& -H_{\varepsilon}\left(\phi_{1}\right)\left(D_{x} \dot{\phi}_{1}+D_{y} \dot{\phi}_{1} p_{j}+D_{y x}^{2} \phi_{1}\left(\dot{h}_{\varepsilon}, \cdot\right)+D_{y y}^{2} \phi_{1}\left(\dot{h}_{\varepsilon}, p_{j}\right)\right)
\end{aligned}
$$

is a map from $X$ to $\mathscr{L}(X, Y)$ and

$$
\begin{aligned}
\mathscr{F}_{5}\left(\varepsilon, p_{j}, q_{j}, q_{l(j)}\right)= & D_{x x}^{2} \dot{\phi}_{2}+D_{x y}^{2} \dot{\phi}_{2}\left(\cdot, p_{j}+H_{\varepsilon}\right)+D_{x x y}^{3} \phi_{2}\left(\cdot, \cdot \dot{h}_{\varepsilon}\right) \\
& +D_{x y y}^{3} \phi_{2}\left(\cdot, \dot{h}_{\varepsilon}, H_{\varepsilon}+p_{j}\right)+D_{y y}^{2} \dot{\phi}_{2}\left(p_{j}, H_{\varepsilon}\right)+D_{y} \dot{\phi}_{2} q_{j} \\
& +D_{y y y}^{3} \phi_{2}\left(\dot{h}_{\varepsilon}, H_{\varepsilon}, p_{j}\right)+D_{y y}^{2} \phi_{2}\left(\dot{h}_{\varepsilon}, q_{j}\right)+D_{y x}^{2} \phi_{2}\left(\dot{H}_{\varepsilon}, \cdot\right) \\
& +D_{y y}^{2} \phi_{2}\left(\dot{H}_{\varepsilon}, p_{j}\right) \\
& -H_{\varepsilon}\left(\phi_{1}\right)\left(D_{x x}^{2} \phi_{1}+D_{x y}^{2} \phi_{1}\left(\cdot, p_{j}+H_{\varepsilon}\right)\right. \\
& +D_{y y}^{2} \phi_{2}\left(H_{\varepsilon}, p_{j}\right)+D_{y} \phi_{1} q_{j}-D_{x} G_{\varepsilon}\left(\phi_{1}\right)\left(\mathscr{A}\left(\varepsilon, h_{\varepsilon}, p_{j}\right), \mathscr{A}_{,} \dot{\phi}_{1}\right. \\
& -G_{\varepsilon}\left(\phi_{1}\right)\left(\mathscr{A}, D_{x} \dot{\phi}_{1}+D_{y} \dot{\phi}_{1} p_{j}+D_{y x}^{2} \phi_{1} \dot{h}_{\varepsilon}+D_{y y}^{2} \phi_{1}\left(\dot{h}_{\varepsilon}, p_{j}\right)\right) \\
& -q_{l(j)}\left(\phi_{1}\right)\left(\dot{\phi}_{1}+D_{y} \phi_{1} \dot{h}_{\varepsilon}, D_{x x}^{2} \phi_{1}\right) \\
& \left.\quad+D_{x y}^{2} \phi_{1}\left(\cdot, p_{j}+H_{\varepsilon}\right)+D_{y y}^{2} \phi\left(p_{j}, H_{\varepsilon}\right)+D_{y} \phi_{1} q_{j}\right) \\
& -q_{j}\left(\phi_{1}\right)\left(\mathscr{A}\left(\varepsilon, h_{\varepsilon}, p_{j}\right), D_{x} \dot{\phi}_{1}+D_{x y}^{2} \phi_{1}\left(\cdot, \dot{h}_{\varepsilon}\right)+D_{y} \dot{\phi}_{1} H_{\varepsilon}\right. \\
& +H_{\varepsilon}\left(\phi_{1}\right)\left(D_{x x}^{2} \dot{\phi}_{1}+D_{x y}^{2} \dot{\phi}_{1}\left(\cdot, p_{j}+H_{\varepsilon}\right)+D_{x x y}^{3} \phi_{1}\left(\cdot, \cdot \dot{h}_{\varepsilon}, H_{\varepsilon}\right)+D_{y} \phi_{1} \dot{H}_{\varepsilon}\right) \\
& +D_{x y y}^{3} \phi_{1}\left(\cdot, \dot{h}_{\varepsilon}, p_{j}+H_{\varepsilon}\right)+D_{y y}^{2} \dot{\phi}_{1}\left(p_{j}, H_{\varepsilon}\right)+D_{y} \dot{\phi}_{1} q_{j} \\
& +D_{y y y}^{3} \phi_{1}\left(p_{j}, \dot{h}_{\varepsilon}, H_{\varepsilon}\right)+D_{y y}^{2} \phi_{1}\left(\dot{h}_{\varepsilon}, q_{j}\right)+D_{y x}^{2} \phi_{1}\left(\dot{H}_{\varepsilon}, \cdot\right) \\
& \left.+D_{y y}^{2} \phi_{1}\left(p_{j}, \dot{H}_{\varepsilon}\right)\right)
\end{aligned}
$$

is a map from $X$ to $\mathscr{L}^{2}(X, Y)$. Furthermore, taking relation (3.8) as it stands and differentiating (3.9) with respect to $\varepsilon$, we obtain the relations $(3.14)_{2}$, where

$$
l(2)=1 \text { and } p_{2}=H_{\varepsilon} .
$$

Note, that here we need the assumption that $f$ and $g$ are of class $C^{3}$.

We shall show that the subspace given by $p_{1}=p_{2}$ and $q_{1}=q_{2}$ is invariant under the flow defined by the system of equations $(3.14)_{1}$ and $(3.14)_{2}$ in $\left(p_{1}, q_{1}, p_{2}, q_{2}\right)$-space. Thus, the identities (3.4) and (3.7) follow, when they are satisfied for $\varepsilon=0$. But this will be a consequence of the initial conditions (3.3) and (3.13).

To summarize, so far we have argued that the problem (3.2) is formally equivalent to an initial-value problem for the differential equation (3.12). Now we are going to justify this argument step by step and to solve the initial-value problem.

We introduce the following notation:

$$
I=\left[-\varepsilon_{0}, \varepsilon_{0}\right]
$$

$$
\mathscr{D}(r, L, M)=\left\{(h, H) \in C_{L}^{1}(X, Y) \times C_{M}^{1}(X, \mathscr{L}(X, Y))\|\| h\left\|_{1} \leqq r,\right\| H\left\|_{0} \leqq r,\right\| H \|_{1} \leqq L\right\}
$$

where $\varepsilon_{0}$ is an arbitrary real number greater than one, and $r, L$, and $M$ are positive constants which are specified later.

LEMMA 3.1. Assume that the conditions of Theorem 2.1 are satisfied and let $\left(h_{\varepsilon}, H_{\varepsilon}\right)=(h, H)$ be any element of $\mathscr{D}(r, L, M)$ where $\mathrm{cl}\left(B_{r}(0)\right) \subset U$. Then, for any $\varepsilon$ in $I$, the equation (3.5) has a unique solution $h_{\varepsilon}=\mathscr{H}_{1}=\mathscr{H}_{1}(\varepsilon, h, H)$ with the following properties:

$$
\mathscr{H}_{1} \in C_{K_{1}}^{1}(X, Y)
$$


where the constant $K_{1}$ can be chosen independently of $L$ and $M$. Furthermore $\left\|\mathscr{H}_{1}\right\|_{0} \leqq r_{1}$ and $\left\|\mathscr{H}_{1}\right\|_{1} \leqq r_{2}$, where

$$
r_{1} \leqq K_{2}\left(\|g\|_{0}+\|f\|_{0}\right), \quad r_{2} \leqq K_{3}\left(\|f\|_{1}+\|g\|_{1}\right),
$$

with some positive constants $K_{2}$ and $K_{3} ; K_{2}$ does not depend on $L$ and $M$. Moreover, $\mathscr{H}_{1}(0)=0\left(\mathscr{H}_{1}(0)=0\right.$ and $\left.D_{x} \mathscr{H}_{1}(0)=0\right)$, provided that $(\mathrm{N} 2)$ and $h(0)=0((\mathrm{~N} 2),(\mathrm{N} 3)$, $h(0)=0, D h(0)=0$, and $H(0)=0$ ) hold.

(ii) The map $(\varepsilon, h, H) \mapsto \mathscr{H}_{1}(\varepsilon, h, H): I \times \mathscr{D}(r, L, M) \rightarrow C^{1}(X, Y)$ is continuous and satisfies a Lipschitz condition with respect to $(h, H)$ with constant $K_{4}$.

Proof. The unique solution of (3.5) is given by

$$
\mathscr{H}_{1}=-\sum_{j=0}^{\infty}\left(\prod_{i=0}^{j} \mathscr{B}^{-1}\left(\phi_{1}^{i}\right)\right) \mathscr{F}_{1}\left(\phi_{1}^{j}\right)
$$

if $(\mathrm{L1})_{4}$ holds, and by

$$
\mathscr{H}_{1}=\sum_{j=0}^{\infty}\left(\prod_{i=1}^{j} \mathscr{B}\left(\phi_{1}^{-i}\right)\right) \mathscr{F}_{1}\left(\phi_{1}^{-j-1}\right)
$$

if $(\mathrm{L} 2)_{4}$ holds. Here we use the estimates $\|\mathscr{A}(\varepsilon, h, D h)-A\|_{0}=O(\delta)$ and $\|\mathscr{B}-B\|_{0}=$ $O(\delta)$ as $\delta \rightarrow 0$. It follows that for sufficiently small $\delta>0$ the map

$$
\mathscr{B}(x): Y \rightarrow Y \quad(\text { where } x \in X) \quad\left(\phi_{1}(\varepsilon, \cdot, h(\cdot)): X \rightarrow X\right)
$$

can be inverted and the estimate $\left\|\mathscr{B}^{-1}-B^{-1}\right\|_{0}=O(\delta)\left(\left\|D_{x} \phi_{1}(\varepsilon, \cdot, h(\cdot))^{-1}-A^{-1}\right\|_{0}=\right.$ $O(\delta))$ holds. Hence,

$$
\left\|\mathscr{B}^{-1}\right\|_{0}\left\|D_{x} \phi_{1}(\varepsilon, \cdot, h(\cdot))\right\|_{0}^{j}<1 \quad\left(\|\mathscr{B}\|_{0}\left\|D_{x} \phi_{1}(\varepsilon, \cdot h(\cdot))^{-1}\right\|_{0}^{j}<1\right)
$$

for all $0 \leqq j \leqq 4$. A straightforward computation shows that the series in $(3.15)((3.16))$ converges in $C^{1}(X, Y)$ and represents a solution of $(3.5)$ for $h_{\varepsilon}$. Uniqueness is easily seen by an a priori $C^{0}$ estimate.

The remaining properties of $\mathscr{H}_{1}$ which are stated in the lemma, are easily seen by inspection of the formulas in (3.15) and (3.16). We simply note that $\left\|F_{1}\right\|_{0} \leqq$ $\tilde{K}_{2}\left(\|g\|_{0}+r\|f\|_{0}\right)$ and $\left\|D_{x} \mathscr{F}_{1}\right\|_{0} \leqq \tilde{K}_{3}\left(\|f\|_{1}+\|g\|_{1}\right)$ holds with some constants $\tilde{K}_{2}$ and $\tilde{K}_{3}$, where $K_{2}$ does not depend on $L$ and $M$. Moreover, $\mathscr{F}_{1}(0)-0$ (resp. $\mathscr{F}_{1}(0)=0$ and $\left.D_{x} \mathscr{F}_{1}(0)=0\right)$ provided that $(\mathrm{N} 2)$ and $h(0)=0($ resp. $(\mathrm{N} 2),(\mathrm{N} 3), h(0)=0, D h(0)=0$, and $H(0)=0$ ) holds. We also remark that Lipschitz constants can be estimated by the sup-norm of derivatives. Since the Lipschitz constant of $D_{x} \mathscr{H}_{1}$ is close to

$$
\left\|D_{x x}^{2} g\right\|_{0}+2\left\|D_{x y}^{2} g\right\|_{0} r+\left\|D_{y y}^{2} g\right\|_{0} r^{2}+\left\|D_{x x}^{2} f\right\|_{0} r^{2}+2\left\|D_{x y}^{2} f\right\|_{0} r^{2}+\left\|D_{y y}^{2} f\right\|_{0} r^{3}
$$

where $\delta$ is sufficiently small, $K_{1}$ can be chosen independently of $L$ and $M$.

To prove continuity of the map in (ii), one uses the fact that each member of the series in (3.15) (resp. (3.16)) has this property and that the convergence is uniform with respect to $(\varepsilon, h, H) \in I \times \mathscr{D}(r, L, M)$.

LemMA 3.2. Suppose that the assumptions of Lemma 3.1 are valid. Then (3.9) has a unique solution $G_{\varepsilon}=\mathscr{G}=\mathscr{G}(\varepsilon, h, H)$ with the following properties:

$$
\mathscr{G} \in C_{K_{s}}^{1}\left(X, \mathscr{L}^{2}(X, Y)\right)
$$

$\|\mathscr{G}\|_{0} \leqq K_{6}$, and $\|\mathscr{G}\|_{1} \leqq K_{7}$, where $K_{5}, K_{6}$ and $K_{7}$ are certain constants; $K_{6}$ does not depend on $L$ and $M$. Furthermore, $\mathscr{G}(0,0,0)=0$.

(ii) The map $(\varepsilon, h, H) \mapsto \mathscr{G}(\varepsilon, h, H): I \times \mathscr{D}(r, L, M) \rightarrow C^{1}\left(X, \mathscr{L}^{2}(X, Y)\right)$ is continuous and satisfies a Lipschitz condition with respect to $(h, H)$ with constant $K_{8}$. Moreover, with $C^{0}\left(X, \mathscr{L}^{2}(X, Y)\right)$ as range, it is continuously differentiable. 
Proof. As in the previous proof, the unique solution of (3.9) is given by

$$
\mathscr{G}=-\sum_{j=0}^{\infty}\left(\prod_{i=0}^{j} \mathscr{B}^{-1}\left(\phi_{1}^{i}\right)\right) \mathscr{F}_{3}\left(\phi_{1}^{j}\right)\left(\prod_{i=j}^{0} \mathscr{A}\left(\phi_{1}^{i}\right), \prod_{i=j-1}^{0} \mathscr{A}\left(\phi_{1}^{i}\right)\right)
$$

if $(\mathrm{L} 1)_{4}$ holds, and by

$$
\mathscr{G}=\sum_{j=0}^{\infty}\left(\prod_{i=1}^{j} \mathscr{B}\left(\phi_{1}^{-1}\right)\right) \mathscr{F}_{3}\left(\phi_{1}^{-j-1}\right)\left(\prod_{i=j+1}^{1} \mathscr{A}^{-1}\left(\phi_{1}^{-i}\right), \prod_{i=j+1}^{1} \mathscr{A}^{-1}\left(\phi_{1}^{-i}\right)\right)
$$

if (L2) ${ }_{4}$ holds. Again, all properties of $G$ which are stated easily follow from these formulae. Note that $\mathscr{F}_{3}(0,0,0)=0$. To see that $K_{6}$ does not depend on $L$ and $M$ we note that

$$
\begin{aligned}
\left\|\mathscr{F}_{3}\right\|_{0} \leqq & |\varepsilon|\left(\left\|D_{x x}^{2} g\right\|_{0}+2\left\|D_{x y}^{2} g\right\|_{0} r+\left\|D_{y y}^{2} g\right\|_{0} r^{2}\right. \\
& \left.+\left\|D_{x x}^{2} f\right\|_{0} r+2\left\|D_{x y}^{2} f\right\|_{0} r^{2}+\left\|D_{y y}^{2} f\right\|_{0} r^{3}\right)
\end{aligned}
$$

LEMMA 3.3. Suppose that the assumptions of the above lemmas hold. Then (3.11), with $\dot{h}_{\varepsilon}=\mathscr{H}_{1}$ from Lemma 3.1 and $G$ from Lemma 3.2 , has a unique solution $H_{\varepsilon}=\mathscr{H}_{2}=$ $\mathscr{H}_{2}(\varepsilon, h, H)$ which has the following properties:

(i) $\quad \mathscr{H}_{2} \in C_{K_{9}}^{1}(X, \mathscr{L}(X, Y)),\left\|\mathscr{H}_{2}\right\|_{0} \leqq r_{3},\left\|\mathscr{H}_{2}\right\|_{1} \leqq K_{10}$

where $r_{3} \leqq K_{11}\left(\|f\|_{1}+\|g\|_{1}\right)$ and $K_{9}, K_{10}, K_{11}$ are certain positive constants; $K_{9}$ can be chosen independently of $M$ and $K_{10}$ independently of $L$ and $M$. Moreover, $\mathscr{H}_{2}(0)=0$ provided that $(\mathrm{N} 2),(\mathrm{N} 3), h(0)=0$, and $H(0)=0$ hold.

(ii) The map $(\varepsilon, h, H) \mapsto \mathscr{H}_{2}(\varepsilon, h, H): I \times \mathscr{D}(r, L, M) \rightarrow C^{1}(X, \mathscr{L}(X, Y))$ is continuous and satisfies a Lipschitz condition with respect to $(h, H)$ with some constant $K_{12}$. Proof. Set

$$
\mathscr{F}_{6}=\mathscr{F}_{6}(\varepsilon, h, H)=\mathscr{F}_{2}(\varepsilon, h, H, \mathscr{G})-\mathscr{C}(\varepsilon, h, H, \mathscr{G}) \mathscr{H}_{1} .
$$

Then the equation which is considered in Lemma 3.3 has a unique solution given by

$$
\mathscr{H}_{2}=\sum_{j=0}^{\infty}\left(\prod_{i=0}^{j} \mathscr{B}^{-1}\left(\phi^{i}\right) \mathscr{F}_{6}\left(\phi_{1}\right) \prod_{i=j}^{0} \mathscr{A}\left(\phi_{1}^{i}\right)\right)
$$

if $(\mathrm{L} 1)_{4}$ holds, and by

$$
\mathscr{H}_{2}=\sum_{j=0}^{\infty}\left(\prod_{i=1}^{j} \mathscr{B}\left(\phi^{-i}\right) \mathscr{H}_{6}\left(\phi^{-j-1}\right) \prod_{i=j+1}^{1} \mathscr{A}^{-1}\left(\phi_{1}^{-i}\right)\right)
$$

if $(\mathrm{L} 2)_{4}$ holds. To prove its stated properties, we note that

$$
\left\|\mathscr{F}_{6}\right\|_{0} \leqq \tilde{K}_{11}\left(\|f\|_{1}+\|g\|_{1}\right)
$$

holds with some constant $\tilde{K}_{11}$. Furthermore,

$$
\begin{aligned}
\left\|D_{x} \mathscr{F}_{6}\right\|_{0} \leq & \left\|D_{x x}^{2} g\right\|_{0}+2\left\|D_{x y}^{2} g\right\|_{0} r+\left\|D_{y y}^{2} g\right\|_{0} r \\
& +\left\|D_{x x}^{2} f\right\|_{0} r+\left\|D_{x y}^{2} f\right\|_{0} r^{2}+\left\|D_{y y}^{2} f\right\|_{0}+O(\delta)
\end{aligned}
$$

the Lipschitz constant of $D_{x} \mathscr{F}_{6}$ with respect to $x$ is smaller than

$$
\begin{aligned}
\left\|D_{x x x}^{3} g\right\|_{0} & +3 r\left\|D_{x x y}^{3} g\right\|_{0}+3 r^{2}\left\|D_{x y y}^{3} g\right\|_{0}+r^{3}\left\|D_{y y}^{3} g\right\|_{0} \\
& +r\left(3 L+|\varepsilon| K_{1}\right)\left\|D_{y y}^{2} g\right\|_{0}+\left(3 L+|\varepsilon| K_{1}\right)\left\|D_{x y}^{2} g\right\|_{0}+r\left\|D_{x x x}^{3} f\right\|_{0} \\
& +3 r^{2}\left\|D_{x x y}^{3} f\right\|_{0}+3 r^{3}\left\|D_{x y y}^{3} f\right\|_{0}+r^{4}\left\|D_{y y y}^{3} f\right\|
\end{aligned}
$$




$$
\begin{gathered}
+r^{2}\left(4 L+\left(L+K_{6}\right)\|\mathscr{A}(\varepsilon, h, H)\|_{0}+|\varepsilon| K_{1}\right)\left\|D_{y y}^{2} f\right\|_{0} \\
+r\left(5 L+2\left(L+K_{6}\right)\|\mathscr{A}(\varepsilon, h, H)\|_{0}+|\varepsilon| K_{1}\right)\left\|D_{y x}^{2} f\right\|_{0} \\
+\left(L+\left(L+K_{6}\right)\|\mathscr{A}(\varepsilon, h, H)\|_{0}\right)\left\|D_{x x}^{2} f\right\|_{0}+O(\delta), \\
\left\|D_{x} \mathscr{A}\right\|_{0} \leqq|\varepsilon|\left(\left\|D_{x x}^{2} f\right\|_{0}+2\left\|D_{x y}^{2} f\right\|_{0} r+\left\|D_{y y}^{2} f\right\|_{0} r^{2}\right)+O(\delta)
\end{gathered}
$$

and

$$
\left\|D_{x} \mathscr{B}\right\|_{0} \leqq|\varepsilon|\left(\left\|D_{x y}^{2} g\right\|_{0}+\left\|D_{y y}^{2} g\right\|_{0} r+\left\|D_{x y}^{2} f\right\|_{0} r+\left\|D_{y y}^{2} f\right\|_{0} r^{2}\right)+O(\delta) \quad \text { as } \delta \rightarrow 0 .
$$

Therefore, $K_{10}$ can be chosen independently of $L$ and $M$, and $K_{9}$ independently of M. Moreover, $\mathscr{F}_{6}(0)=0$, provided that $(\mathrm{N} 2),(\mathrm{N} 3), h(0)=0$ and $H(0)=0$ holds. But this implies $\mathscr{H}_{2}(0)=0$. The rest of the proof is similar to the previous proofs.

By Lemma 3.1 and Lemma 3.3, the right-hand side of the differential equation (3.12) is given by

$$
\mathscr{H}(\varepsilon, h, H)=\left(\begin{array}{l}
\mathscr{H}_{1}(\varepsilon, h, H) \\
\mathscr{H}_{2}(\varepsilon, h, H)
\end{array}\right) .
$$

It is uniquely determined by the stated properties. Next we are going to solve this equation with initial values $h=0$ and $H=0$ at $\varepsilon=0$. To this end we select $r, L$ and $M$ such that

$$
\mathrm{cl}\left(B_{r}(0)\right) \subset U, L \geqq \varepsilon_{0} \max \left(K_{1}, K_{10}\right) \quad \text { and } \quad M \geqq \varepsilon_{0} K_{9}(L) .
$$

We also assume that the conditions

$$
r_{i} \leqq \frac{r}{\varepsilon_{0}} \quad(i=1,2,3)
$$

are valid. This is achieved by requiring $\delta$ to be sufficiently small since $r_{i}=O(\delta)$ as $\delta \rightarrow 0$. Note that $r$ has been chosen independently of $\delta$. On the other hand we point out that $\mathrm{cl}\left(B_{\varepsilon_{0} r_{1}}(0)\right) \subset U$ has to be true, but not necessarily $\mathrm{cl}\left(B_{r}(0)\right) \subset U$. Hence, under certain circumstances one can shrink $U$ to make $\delta$ sufficiently small and work with functions $h$ such that $h(x) \in U$ for all $x \in X$.

LeмmA 3.4. Let the assumptions of Theorem 2.1 be true. Furthermore, suppose that the constants $r, L, M$ and $\delta$ are chosen such that the conditions (I1) and (I2) are fulfilled. Then the differential equation (3.12) has a unique solution $\varepsilon \mapsto\left(h_{\varepsilon}(x), H_{\varepsilon}(x)\right)$ in the interval $I$, which has the following properties:

(i) $\varepsilon \mapsto h_{\varepsilon} \in C^{1}\left(I, C_{L}^{1}(X, Y)\right), \varepsilon \mapsto H_{\varepsilon} \in C^{1}\left(I, C^{1}(X, \mathscr{L}(X, Y))\right)$, where $\left(h_{\varepsilon}, H_{\varepsilon}\right) \in$ $\mathscr{D}(r, L, M)$ holds for all $\varepsilon$ in $I$, and $h_{0}(x)=0, H_{0}(x)=0$ for all $x \in X$. Moreover, $h_{\varepsilon}(0)=0$ $\left(h_{\varepsilon}(0)=0, D_{x} h_{\varepsilon}(0)=0\right.$ and $\left.H_{\varepsilon}(0)=0\right)$ for all $\varepsilon$ in $I$, provided that $(\mathrm{N} 2)((\mathrm{N} 2)$ and $(\mathrm{N} 3))$ holds.

(ii) $D_{x} h_{\varepsilon}=H_{\varepsilon}, D_{x} H_{\varepsilon}=G_{\varepsilon}=\mathscr{G}\left(\varepsilon, h_{\varepsilon}, H_{\varepsilon}\right)$.

Proof. The proof of part (i) follows the lines of the proof of the usual PicardLindelof theorem for ordinary differential equations. We look for a continuous solution $\varepsilon \mapsto\left(h_{\varepsilon}, H_{\varepsilon}\right)$ of the integral equation

$$
\left(\begin{array}{c}
h_{\varepsilon} \\
H_{\varepsilon}
\end{array}\right)=\int_{0}^{\varepsilon} \mathscr{H}\left(\sigma, h_{\sigma}, H_{\sigma}\right) d \sigma \quad(\varepsilon \in I) .
$$

This problem is equivalent to solving the initial-value problem (3.12), (3.3), and (3.13). In particular, a continuous solution of (3.21) is continuously differentiable. According 
to the previous lemmas, the right-hand side of (3.21) defines a contraction map $\mathscr{T}$ of the metric space

$$
\begin{aligned}
S= & \left\{\varepsilon \mapsto\left(h_{\varepsilon}, H_{\varepsilon}\right) \in C^{0}\left(I, C_{L}^{1}(X, Y)\right)\right. \\
& \left.\times C^{0}\left(I, C_{M}^{1}(X, \mathscr{L}(X, Y))\right) \mid\left(h_{\varepsilon}, H_{\varepsilon}\right) \in \mathscr{D}(r, L, M) \text { for all } \varepsilon \in I\right\}
\end{aligned}
$$

into itself, where the metric is given by the norm defined by

$$
\sup _{\varepsilon \in I}\left(e^{\gamma|\varepsilon|}\left\|h_{\varepsilon}\right\|_{1}, e^{\gamma|\varepsilon|}\left\|H_{\varepsilon}\right\|_{1}\right)
$$

with

$$
\gamma>\max \left(K_{2}, K_{12}\right) .
$$

Obviously, with this metric $S$ is complete. Therefore $\mathscr{T}$ has a unique fixed point in $S$, which is the desired solution. Moreover, the set

$$
S_{0}=\left\{\varepsilon \mapsto\left(h_{\varepsilon}, H_{\varepsilon}\right) \in S \mid h_{\varepsilon}(0)=0 \text { for all } \varepsilon \in I\right\}
$$

or $S_{1}=\left\{\varepsilon \mapsto\left(h_{\varepsilon}, H_{\varepsilon}\right) \in S \mid h_{\varepsilon}(0)=0, D_{x} h_{\varepsilon}(0)=0\right.$ and $\left.H_{\varepsilon}(0)=0\right\}$ for all $\varepsilon \in I$ is closed and invariant under the map $\mathscr{T}$, provided that (N2) (resp. (N2) and (N3)) holds. Hence, the unique fixed point of $\mathscr{T}$ in $S$ lies in $S_{0}\left(S_{1}\right)$. Thus, part (i) is proved.

To prove (ii), let $\left(h_{\varepsilon}, H_{\varepsilon}\right)$ be given by the solution of (3.21) constructed above and define $G_{\varepsilon}$ to be $\mathscr{G}\left(\varepsilon, h_{\varepsilon}, H_{\varepsilon}\right)$ for $\varepsilon$ in $I$. Note that $p_{1}(\varepsilon)=D_{x} h_{\varepsilon}, q_{1}(\varepsilon)=D_{x} H_{\varepsilon}$, $p_{2}(\varepsilon)=H_{\varepsilon}$ and $q_{2}(\varepsilon)=G_{\varepsilon}$ defines a solution of the system of equations in (3.14) 1 and $(3.14)_{2}$. These relations are fulfilled in the space $\left(C^{0}\left(I, C^{0}(X, \mathscr{L}(X, Y))\right) \times\right.$ $\left.C^{0}\left(I, C^{0}\left(X, \mathscr{L}^{2}(X, Y)\right)\right)\right)^{2}$. Furthermore, by $(3.3),(3.13)$ and $\mathscr{G}(0,0,0)=0$,

$$
p_{1}(0)=p_{2}(0)=0, \quad q_{1}(0)=q_{2}(0)=0
$$

holds for this solution. Therefore it remains to show that this implies $p_{1}(\varepsilon)=p_{2}(\varepsilon)$ and $q_{1}(\varepsilon)=q_{2}(\varepsilon)$ for all $\varepsilon \in I$.

Using the formulas (3.19) (resp. (3.20)) and (3.17) (resp. (3.18)), we can rewrite the relations in $(3.14)_{j}(j=1,2)$ in the form

$$
\dot{p}_{j}(\varepsilon)=\mathscr{H}_{3}\left(\varepsilon, p_{j}(\varepsilon), q_{j}(\varepsilon)\right), \quad q_{j}(\varepsilon)=\mathscr{H}_{4}\left(\varepsilon, p_{j}(\varepsilon), q_{j}(\varepsilon), q_{l(j)}(\varepsilon)\right)
$$

where the maps $\varepsilon \mapsto \mathscr{H}_{3}\left(\varepsilon, p_{j}(\varepsilon), q_{j}(\varepsilon)\right): I \rightarrow C^{0}(X, \mathscr{L}(X, Y))$ and $\varepsilon \rightarrow \mathscr{H}_{4}\left(\varepsilon, p_{j}(\varepsilon)\right.$, $\left.q_{j}(\varepsilon), q_{l(j)}(\varepsilon)\right)$ are continuous, and

$$
\begin{aligned}
& \left\|\mathscr{H}_{3}\left(\varepsilon, p_{1}(\varepsilon), q_{1}(\varepsilon)\right)-\mathscr{H}_{3}\left(\varepsilon, p_{2}(\varepsilon), q_{2}(\varepsilon)\right)\right\|_{0} \leqq K_{13} \alpha(\varepsilon), \\
& \left\|\mathscr{H}_{4}\left(\varepsilon, p_{1}(\varepsilon), q_{1}(\varepsilon), q_{2}(\varepsilon)\right)-\mathscr{H}_{4}\left(\varepsilon, p_{2}(\varepsilon), q_{2}(\varepsilon), q_{1}(\varepsilon)\right)\right\|_{0} \leqq K_{13} \alpha(\varepsilon),
\end{aligned}
$$

holds for all $\varepsilon$ in $I$ with some positive constant $K_{13}$ and

$$
\alpha(\varepsilon)=\sup \left(\left\|p_{1}(\varepsilon)-p_{2}(\varepsilon)\right\|_{0},\left\|q_{1}(\varepsilon)-q_{2}(\varepsilon)\right\|_{0}\right) .
$$

Integrating the equations in $(3.23)_{j}$ from 0 to $\varepsilon$, subtracting the integral relations which are obtained for $j=1$ and $j=2$ and using (3.22), we get the following estimate

$$
\alpha(\varepsilon) \leqq\left|\int_{0}^{\varepsilon} K_{13} \alpha(\sigma) d \sigma\right| \quad(\varepsilon \in I) .
$$

Gronwall's lemma yields $\alpha(\varepsilon)=0$ for all $\varepsilon$ in $I$, i.e., $p_{1}(\varepsilon)=p_{2}(\varepsilon)$ and $q_{1}(\varepsilon)=q_{2}(\varepsilon)$.

Taking the map $h_{\varepsilon}$ which has been constructed in Lemma 3.4 and setting $\varepsilon=1$, the existence part of Theorem 2.1 follows according to the discussion previous to the above lemmas. 
The proof also yields uniqueness, but only within the class of families of maps $h_{\varepsilon}$ (where $\varepsilon \in I$ ) which have the properties stated in Lemma 3.4. To prove the uniqueness assertion of Theorem 2.1 we therefore have to give a different argument. Here we can even weaken our assumptions considerably.

LEMMA 3.5. Suppose the maps $f \in C^{0}(X \times U, X)$ and $g \in C^{0}(X \times U, Y)$ satisfy a Lipschitz condition with respect to $y$ with constant $\delta>0$. Furthermore, assume that $\left\|B^{-1}\right\|<1$ (resp. $\left.\|B\|<1\right)$ ) holds. Then for each $\varepsilon$ in $1,(3.2)$ has at most one solution $h_{\varepsilon}=h \in C_{L}^{0}(X, Y)$ (resp. such that the map $x \mapsto \phi_{1}(\varepsilon, x, h(x)): X \rightarrow X$ is surjective), where

$$
L<\left(\left\|B^{-1}\right\|^{-1}-1-\delta \varepsilon\right) /(\delta \varepsilon) \quad(\text { resp. } L<(1-\|B\|-\delta \varepsilon) /(\delta \varepsilon)) .
$$

Proof. Assume that $h$ and $\tilde{h}$ are two such solutions of equation (3.2). Then,

$$
h-\tilde{h}=B^{-1}\left(h\left(\phi_{1}\right)-\tilde{h}\left(\phi_{1}\right)+\tilde{h}\left(\phi_{1}\right)-\tilde{h}\left(\phi_{1}(\varepsilon, \cdot \tilde{h}(\cdot))\right)+\varepsilon g(\cdot, \tilde{h}(\cdot))-\varepsilon g(\cdot, h(\cdot))\right)
$$

$\left(\right.$ resp. $\left.h\left(\phi_{1}\right)-\tilde{h}\left(\phi_{1}\right)=B(h-\tilde{h})+\tilde{h}\left(\phi_{1}(\varepsilon, \cdot \tilde{h}(\cdot))\right)-\tilde{h}\left(\phi_{1}\right)+\varepsilon g(\cdot, h(\cdot))-\varepsilon g(\cdot, \tilde{h}(\cdot))\right)$,

in which we use our standing convention that $\phi_{1}=\phi_{1}(\varepsilon, \cdot, h(\cdot))$. Thus by the assumptions

$$
\begin{aligned}
& \|h-\tilde{h}\|_{0} \leqq\left\|B^{-1}\right\|(1+\delta \varepsilon L+\delta \varepsilon)\|h-\tilde{h}\|_{0}<\|h-\tilde{h}\|_{0} \\
& \left(\operatorname{resp} .\|h-\tilde{h}\|_{0} \leqq(\|B\|+\delta \varepsilon L+\delta \varepsilon)\|h-\tilde{h}\|_{0}<\|h-\tilde{h}\|_{0}\right)
\end{aligned}
$$

follows, which implies $h=\tilde{h}$.

Remark 3.6. The initial-value problem (3.12), (3.3) and (3.13) also has a unique solution in a ball around the origin in the space $C_{L}^{0}(X, Y) \times C_{M}^{0}(X, \mathscr{L}(X, Y))$ with appropriate constants $L$ and $M$, even under the weaker assumptions that $f$ and $g$ are of class $C_{\mathrm{Lip}}^{2}$ and that $(\mathrm{L} 1)_{3}$ or $(\mathrm{L} 2)_{3}$ holds. However, it is not obvious how to show $D_{x} h_{\varepsilon}=H_{\varepsilon}$, to make sure that the solution actually yields a solution of (3.2).

On the other hand, one can still use the deformation principle to prove existence of a $C_{\text {Lip }}^{2}$ invariant manifold under the weaker assumptions mentioned above. This requires the solution of the nonlinear equation (3.6) for $H_{\varepsilon}$ in some space $C_{M}^{1}(X, \mathscr{L}(X, Y))$ as a Lipschitz continuous function of $\varepsilon$ in $I$ and $h_{\varepsilon} \in C_{L}^{1}(X, Y)$. Here the identity $D_{x} h_{\varepsilon}=H_{\varepsilon}$ follows from the fact that $D_{x} h_{\varepsilon}$ as well as $H_{\varepsilon}$ are solutions of the first equation in (3.14) for $p_{j}$, if we set $q_{j}=D_{x} H_{\varepsilon}$. In general, one does not have an explicit representation for the solution of (3.6); one can, however, use the contraction mapping principle to solve it. Thus, this method is a combination of the usual fixed point method to construct invariant manifolds [15] and the pure deformation method which we have proposed in the present paper.

4. Proof of Corollary 2.2. Corollary 2.2 is a consequence of Theorem 2.1 together with the following lemma; a bootstrapping argument accomplishes our purpose.

LeMma 4.1. (a) Assume that $f \in C^{k}(X \times U, X)$ and $g \in C^{k}(X \times U, Y)$ holds for some $k \geqq 3$. Furthermore, let $(\mathrm{L} 1)_{k}$ or $(\mathrm{L} 2)_{k},\|f\|_{1}<\delta$, and $\|g\|_{1}<\delta$ hold. Suppose that for fixed $\varepsilon, h_{\varepsilon}=h \in C^{k-1}(X, Y)$ is a solution of $(3.2)$. Then $h \in C^{k}(X, Y)$ if $\delta$ is sufficiently small, generally depending on $k$ and $\|h\|_{1}$ for fixed $A$ and $B$.

(b) If $f$ and $g$ are of class $C_{\mathrm{Lip}}^{k}$ for some $k \geqq 2$ and $(\mathrm{L} 1)_{k+1}$ or $(\mathrm{L} 2)_{k+1},\|f\|_{1}<\delta$, and $\|g\|_{1}<\delta$ holds, then any $C^{k}$ solution of $(3.2)$ is contained in $C_{\mathrm{Lip}}^{k}(X, Y)$ for sufficiently small $\delta>0$. 
Proof. Since $h$ is at least of class $C^{2}$ and a solution of (3.3), by uniqueness of the solution of (3.9) we have $D_{x x}^{2} h=\mathscr{G}\left(\varepsilon, h, D_{x} h\right)$ with $\mathscr{G}$ given either by (3.17) or by (3.18). Hence, it remains to show that $h \in C^{k-1}(X, Y)$ implies $\mathscr{G}\left(\varepsilon, h, D_{x} h\right) \in$ $C^{k-2}\left(X, \mathscr{L}^{2}(X, Y)\right)$ in case (a), and $h \in C^{k}(X, Y)$ implies $\mathscr{G}\left(\varepsilon, h, D_{x} h\right) \in$ $C_{\text {Lip }}^{k-2}\left(X, \mathscr{L}^{2}(X, Y)\right)$ in case (b).

If $f$ and $g$ are of class $C^{k}$ and $h \in C^{k-1}(X, Y)$ for some $k \geqq 2$, then the $(k-2)$ nd derivative of each term in the series (3.17) (resp. (3.18)) exists and is continuous. It is easily proved by induction with respect to $k$ that these derivatives are of the form $(j=0,1,2, \cdots)$

$$
\begin{aligned}
& \sum_{\Sigma \alpha=k-2}\left(\prod_{i=0}^{j} D_{x}^{\alpha} \mathscr{B}^{-1}\left(\phi_{1}^{i}\right) \mathscr{P}_{\alpha i}\right) D_{x}^{\alpha} \mathscr{F}_{3}\left(\phi_{1}^{j}\right) \mathscr{P}_{\alpha j} \\
& \cdot\left(\prod_{i=j-1}^{0} D_{x}^{\alpha} \mathscr{A}\left(\phi_{1}^{i}\right) \mathscr{P}_{\alpha i}, \prod_{i=j-1}^{0} D_{x}^{\alpha} \mathscr{A}\left(\phi_{1}^{-i}\right) \mathscr{P}_{\alpha i}\right)
\end{aligned}
$$

or

where

$$
\begin{aligned}
\sum_{\Sigma \alpha=k-2}\left(\prod_{i=1}^{j} D_{x}^{\alpha} \mathscr{B}\left(\phi_{1}^{-i}\right) \mathscr{P}_{\alpha i}\right) D_{x}^{\alpha} \mathscr{F}_{3}\left(\phi^{-j-1}\right) \mathscr{P}_{\alpha(j+1)} \\
\cdot\left(\prod_{i=j+1}^{1} D_{x}^{\alpha} \mathscr{A}^{-1}\left(\phi_{1}^{-i}\right) \mathscr{P}_{\alpha i}, \prod_{i=j+1}^{1} D_{x}^{\alpha} \mathscr{A}^{-1}\left(\phi_{1}^{-i}\right) \mathscr{P}_{\alpha i}\right)
\end{aligned}
$$

$$
\mathscr{B}=\mathscr{B}\left(\varepsilon, h, D_{x} h\right), \quad \mathscr{F}_{3}=\mathscr{F}_{3}\left(\varepsilon, h, D_{x} h\right), \quad \mathscr{A}=\mathscr{A}\left(\varepsilon, h, D_{x} h\right),
$$

and $\mathscr{P}_{\alpha i}$ is an $\alpha$-tuple of products with $i$ factors of the form

$$
\begin{aligned}
& D_{x}^{\alpha} \mathscr{A}\left(\phi_{1}^{n}\right) \mathscr{P}_{\alpha n} \quad(0 \leqq n \leqq i-1) \\
& \text { (resp. } \left.D_{x}^{\alpha} \mathscr{A}^{-1}\left(\phi_{1}^{-n}\right) \mathscr{P}_{\alpha n} \quad(1 \leqq n \leqq i)\right) .
\end{aligned}
$$

These sums have less than $\frac{1}{2} k !(j+1)^{k-2}$ terms, each of which is a "product" of less than $(k+1) j+2$ (resp. $(k+1)(j+1)$ ) factors, with at least $j-k+3$ (resp. $j-k+2)$ factors $\mathscr{B}^{-1}$ (resp. $\left.\mathscr{B}\right)$, at most $k j($ resp. $k(j+1))$ factors $\mathscr{A}\left(\right.$ resp. $\left.\mathscr{A}^{-1}\right)$. Besides these factors there are at most $k-2$ factors which are derivatives of such factors, or of $\mathscr{F}_{3}$ of order less than or equal to $k-2$. Of course, $\mathscr{F}_{3}$ is itself a factor if no derivative of it is contained in the product.

Now assume that

$$
\left.\left\|\mathscr{B}^{-1}\right\|_{0}\|\mathscr{A}\|_{0}^{i}<q<1 \quad \text { (resp. }\|\mathscr{B}\|_{0}\left\|\mathscr{A}^{-1}\right\|_{0}^{i}<q<1\right) \quad(0 \leqq i \leqq k)
$$

holds, where $q$ is some real number which does not depend on $i$. Then

$$
K_{14}(j+1)^{k-2} q^{j-k+3} \quad(j>k-3) \quad\left(\text { resp. } K_{14}(j+1)^{k-2} q^{j-k+2}(j>k-2)\right)
$$

is an upper bound for the $C^{0}$ norm of the sum in (4.1) (resp. (4.2)), where the constant $K_{14}$ depends on $k$. Consequently, the series of these sums over $j$ converges uniformly with respect to $x$ and represents the $(k-2)$ nd derivative of $\mathscr{G}\left(\varepsilon, h, D_{x} h\right)$. But, by $(\mathrm{L} 1)_{k}$ (resp. $\left.(\mathrm{L} 2)_{k}\right),(4.3)_{k}$ is satisfied for some number $q$, provided that $\delta$ is sufficiently small. Thus, part (a) of Lemma 4.1 follows.

Under the assumptions of part (b) the sum in (4.1) (resp. (4.2)) is contained in $C_{\text {Lip }}^{0}\left(X, \mathscr{L}^{k}(X, Y)\right)$ for each $j$. Furthermore, if $(4.3)_{k+1}$ holds, by the above information about this sum, its Lipschitz constant $L_{j}$ can be estimated from above by

$$
K_{15}(j+1)^{k-1} q^{j-k+2} \quad(j>k-2) \quad\left(\text { resp. } K_{15}(j+1)^{k-1} q^{j-k+1}(j>k-1)\right)
$$


with some constant $K_{15}$ that depends on $k$. But by (L1) $)_{k+1}(\operatorname{resp}$. (L2) $k+1$ ), the condition $(4.3)_{k+1}$ is satisfied for sufficiently small $\delta>0$. It follows that $D_{x}^{k-2} \mathscr{G}\left(\varepsilon, h, D_{x} h\right) \in$ $C_{\text {Lip }}^{0}\left(X, \mathscr{L}^{k}(X, Y)\right)$, since $\sum_{j=0}^{\infty} L_{j}<\infty$. Thus, part (b) of Lemma 4.1 holds.

Remark 4.2. In case of center manifolds, the $C_{\text {Lip }}^{k}$ result, even for $k=0$ and $k=1$, is the usual result which is obtained by a fixed point argument ([15], [18]). For $k=0$ one assumes that $(\mathrm{L} 1)_{1}$ or $(\mathrm{L} 2)_{1}$ holds and that $\|f\|_{0},\|g\|_{0}$, and the Lipschitz constants for $f$ and $g$ are sufficiently small. For $k \geqq 1$ the assumptions are analogous to those of Theorem 2.1. For $k=2$, see also Remark 3.6.

The $C_{\text {Lip }}^{2}$ center-manifold theorem together with Lemma 4.1(a) now yields the center manifold theorem in $C^{k}$ spaces for any $k \geqq 3$.

Moreover, observe that for fixed $h_{\varepsilon}$ in $C^{1}(X, Y)$, (3.6) can be solved for $H_{\varepsilon}$ in the space $C^{1}(X, \mathscr{L}(X, Y))$, provided that $f$ and $g$ are of class $C^{2},\|f\|_{1}$ and $\|g\|_{1}$ are sufficiently small, and (L1) $)_{2}$ or (L2) $)_{2}$ holds (cf., Remark 3.6). Thus it follows that the $C_{\text {Lip }}^{1}$ center manifold is actually contained in the class $C^{2}$ in this case; but this is the $C^{2}$ center manifold theorem.

Acknowledgments. We thank Ethan Akin, Marty Golubitsky, Jack Hale, Morris Hirsch, Pat McSwiggen and Charles Pugh for their comments.

\section{REFERENCES}

[1] R. Abraham, J. Marsden and T. Ratiu, Manifolds, Tensor Analysis and Application, AddisonWesley, London, 1983, 2nd ed., Springer-Verlag, New York, to appear.

[2] H. AMANN, Gewöhnliche Differentialgleichugen, Walter de Gruyter-Verlag, Berlin, 1983.

[3] J. CARR, Applications of Center Manifold Theory, Springer-Verlag, New York, 1981.

[4] S. N. Chow ANd J. Hale, Methods of Bifurcation Theory, Springer-Verlag, New York, 1982.

[5] E. A. Coddington And N. Levinson, Theory of Ordinary Differential Equations, McGraw-Hill, New York, 1955.

[6] N. FENICHEL, Persistence and smoothness of invariant manifolds for flows, Indiana Univ. Math. J., 21 (1971), pp. 193-226.

[7] J. HAdAmARD, Sur l'iteration et les solutions asymptotiques des équations differentielles, Bull. Soc. Math. France, 29 (1901), pp. 224-228.

[8] J. HALE, Ordinary Differential Equations, John Wiley, New York, 1969.

[9] J. HAle AND J. SChEURLe, Smoothness of bounded solutions of nonlinear evolution equations, J. Differential Equations, 56(1) (1985), pp. 142-163.

[10] P. Hartman, Ordinary Differential Equations, 2nd ed., Birkhäuser--Verlag, Boston, 1982.

[11] M. M. Hirsch, C. C. PUGH And M. SHub, Invariant Manifolds, Lecture Notes in Mathematics 583, Springer-Verlag, Berlin, 1977; see also M. M. HiRsCH AND C. PUGH, Stable manifolds and hyperbolic sets, Proc. Symp. Pure Math., 14 (1970), pp. 133-164.

[12] M. C. IRwIN, Smooth Dynamical Systems, Academic Press, New York, 1980; see also, On the stable manifold theorem, Bull. London Math. Soc., 2 (1970), pp. 196-198; and On the smoothness of the composition map, Quart. J. Math., Oxford Ser. (2) 23 (1972), pp. 113-133.

[13] A. KÄLLEN, On the proof of the centre manifold theorem, J. London Math. Soc., II, Ser. 26 (1982), pp. 169-173.

[14] A. KelleY, The stable, center-stable, center, center-unstable and unstable manifolds, J. Differential Equations, 3 (1967), pp. 546-570.

[15] J. MARSDEN AND M. MCCRACKen, The Hopf bifurcation and its applications, Applied Mathematical Science, Vol. 19, Springer-Verlag, New York, 1976.

[16] D. Ruelle And F. TAKens, On the nature of turbulence, Comm. Math. Phys., 20 (1971), pp. 167-192.

[17] R. J. SACKER, A new approach to the perturbation theory of invariant surfaces, Comm. Pure Appl. Math., 18 (1965), pp. 717-732.

[18] K. R. SCHNEIDER, On quasicentre manifolds of semilinear equations in Banach spaces, Math. Nachr., 122 (1985), pp. 215-229.

[19] J. SiJBRAND, Studies in nonlinear stability and birfurcation theory, Ph.D. thesis, Univ. of Utrecht, The Netherlands, 1981.

[20] P. MCSWIGGeN, private communication. 